\title{
A SURVEY OF PARABENS IN COMMERCIAL BABY WIPES FROM BRAZIL AND ESTIMATION OF DAILY EXPOSURE
}

\author{
Bruno Alves Rocha, ${ }^{\mathrm{a}, \mathrm{b}, *,(1)}$, Mariana Zuccherato Bocato ${ }^{\mathrm{a}}$, Elder Francisco Latorraca ${ }^{\mathrm{c}}$, João Paulo Bianchi Ximenez ${ }^{\mathrm{a}}$ and \\ Fernando Barbosa Jr. ${ }^{a}$ \\ aDepartamento Análises Clínicas, Toxicológicas e Bromatológicas, Faculdade de Ciências Farmacêuticas de Ribeirão Preto, \\ Universidade de São Paulo, 14040-903 Ribeirão Preto - SP, Brasil \\ ' Instituto de Ciências Ambientais, Químicas e Farmacêuticas, Universidade Federal de São Paulo, 09972-270 Diadema - SP, Brasil \\ 'Núcleo de Ciências da Saúde, Universidade do Estado de Minas Gerais, 37900-106 Passos - MG, Brasil
}

Recebido em 12/11/2019; aceito em 07/01/2020; publicado na web em 12/03/2020

\begin{abstract}
Several studies have shown endocrine-disrupting effects of parabens. Parabens are a group of antimicrobials used as preservatives in a wide variety of products, mainly in personal care products. However, little information is available regarding the occurrence of parabens in baby products. In this study, the concentrations of five commonly used parabens, methyl-, ethyl-, propyl-, butyl- and, benzyl-paraben in 50 Brazilian baby wet wipes were analyzed by liquid chromatography coupled to mass spectrometry. All samples contained parabens, and the total concentrations ranged from 16 to 332,470 $\mathrm{ng} \mathrm{g}^{-1}$ (geometric mean: $5344 \mathrm{ng} \mathrm{g}^{-1}$ ). Methyl-paraben, ethyl-paraben, propyl-paraben, and butyl-paraben were found in $>65 \%$ of the samples. 24 out of 50 samples exceed the permitted value of $0.4 \%$ for methyl-paraben for their use in personal care products. Although, propyl-paraben and butyl-paraben are forbidden in cosmetic products designed for application on the nappy area of children under three years of age, the detection rate of them were 94 and $70 \%$, respectively. Based on the measured concentrations and use frequency of baby wipes the estimated daily intake of parabens was calculated. The median estimated daily intake values of total concentrations of parabens for babies of one year old was $391 \mathrm{ng} \mathrm{kg}^{-1}$ bw day ${ }^{-1}$. In conclusion, babies and children may be exposed to high levels of parabens through use of baby wipes. It is also worth to mention that two forbidden parabens were frequently detected.
\end{abstract}

Keywords: parabens; preservatives; endocrine-disrupting chemicals; baby wipes; estimated daily intake.

\section{INTRODUCTION}

Endocrine disruptors constitute a group of chemical compounds, of natural or synthetic origin, that have been gaining a great prominence in the areas of toxicology and environmental health, for which exposure may lead alterations in the normal functions of the endocrine system, causing possible adverse health effects. ${ }^{1-7}$ Among them, we can cite the parabens, widely used preservative chemicals. Parabens are alkyl esters derived from p-hydroxybenzoic acid and their bio- and physico-chemical properties such as water solubility, stability and antimicrobial activity make them interesting additives in consumer products. ${ }^{1,8-10}$ Over the recent years the concern over the human exposure to paraben-containing products has raised mainly due to their potential endocrine-disrupting effects on the hormonal system. $^{11-13}$

Dermal absorption is the main source of exposure to parabens and personal care products account for about $66 \%$ of this exposure. ${ }^{9,10,13-17}$ After human exposure, the parabens penetrate the systemic circulation and are rapidly biotransformed in the liver. The rapid metabolism of parabens suggests that they are metabolized faster after the intake than by dermal absorption, thus explaining that topical use is the main source of exposure to parabens. ${ }^{18-21}$ Parabens are rapidly absorbed through the gastrointestinal tract or, more slowly, through the skin because the latter mechanism depends on the action of carboxylesterase enzymes and subcutaneous fat that affect the dermal absorption. ${ }^{22}$ After absorption or ingestion, metabolism and urinary excretion are influenced by the size of the paraben alkyl chain, giving them short half-lives in the human body, ranging from 22 minutes for methylparaben to 87 minutes for butylparaben. ${ }^{23}$
Decrease in sperma production, complications in the couple's fecundity, breast tumors and melanomas have been observed in the presence of parabens in biological models and epidemiologic studies. ${ }^{24-27}$ Hormones control the normal development of tissues from the fertilized sperm and egg to the fully developed of human. When a tissue is developing, it is more sensitive to the action of hormones and thus endocrine disruptors, such as, parabens. Moreover, children's metabolic pathways are immature. This means that children's ability to metabolize and excrete those compounds is lower than that of adults', making them more vulnerable to these chemicals. ${ }^{28}$ Therefore, regulatory agencies in several countries banned the use of some parabens in baby wipes ${ }^{29}$ or reduced the maximum percentage allowed in cosmetic formulations, for example, as in the European Union a maximum concentration of $0.14 \%$ was established for the use of $\mathrm{PrP}$ and BuP when used alone or in mixtures with other esters. ${ }^{30}$

Due to direct contact, some quick-clean personal hygiene products such as adult personal hygiene wet wipes and baby wipes become facilitators of human exposure to parabens, ${ }^{31}$ triggering, even, other additional pathologies like allergic processes and anogenital dermatitis. ${ }^{32}$ So, the present study aimed to determine concentrations of five parabens (methylparaben and propylparaben, the most frequently used paraben compounds and, ethylparaben, butylparaben, and benzylparaben, important synthetic compounds in the paraben family) in 50 commercial baby personal hygiene wet wipes collected from Brazil and estimating the exposure doses via dermal contact.

\section{EXPERIMENTAL}

\section{Chemicals and materials}

Methyl-paraben (methyl 4-hydroxybenzoate; MeP), ethyl-paraben 
(ethyl 4-hydroxybenzoate; EtP), propyl-paraben (propyl 4-hydroxybenzoate PrP), butyl-paraben (butyl 4-hydroxybenzoate; BuP), benzyl-paraben (benzyl 4-hydroxybenzoate; BzP) and parabens internal standard mix solution (methyl 4-hydroxybenzoate-ring- ${ }^{13} \mathrm{C}_{6}$, ethyl 4-hydroxybenzoate-ring- ${ }^{13} \mathrm{C}_{6}$, propyl 4-hydroxybenzoatering- ${ }^{13} \mathrm{C}_{6}$, butyl 4-hydroxybenzoate-ring- ${ }^{13} \mathrm{C}_{6}$ ) were acquired from Sigma-Aldrich (St. Louis, MO, USA). HPLC grade methanol was purchased from JT Baker (Phillipsburg, NJ, USA). Ultrapure water used throughout the experiment was obtained from Milli-Q water purification system (Millipore RiOs-DITM, Bedford, MA, USA). The individual stock solutions of each compound and internal standards were prepared by dissolution in methanol and stored in polypropylene capped tube at $-20^{\circ} \mathrm{C}$. The calibration and working standard solutions were prepared daily from the stock solutions through serial dilution with methanol:water (1:1), and stored polypropylene capped tube at $-20{ }^{\circ} \mathrm{C}$ until LC-MS/MS analysis.

\section{Instrumentation and sample analysis}

The analysis was performed with a Thermo Scientific LC system equipped with a pump (Accela 600 pump) and an autosampler coupled with Thermo Scientific TSQ Quantum Access Max with an electrospray triple quadrupole mass spectrometer (Thermo Scientific $^{\mathrm{TM}}$, San Jose, CA, USA). The chromatographic separation was carried out on an Atlantis ${ }^{\circledR} \mathrm{T} 3 \mathrm{dC} 18$ column $(75 \mathrm{~mm}$ x $2.1 \mathrm{~mm}$ i.d. and $3.0 \mu \mathrm{m}$ particle size, Waters, Milford, MS, USA). A gradient program for the mobile phase was set as the following: mobile phase A was water, and mobile phase $\mathrm{B}$ was methanol. A gradient program was used with a total run time of $10 \mathrm{~min}$ : $0.00-6.50 \mathrm{~min} 25-95 \% \mathrm{~B}$; $6.51-8.00 \min 95 \% \mathrm{~B} ; 8.01-8.20 \min 25 \% \mathrm{~B} ; 8.21-10.00 \min 25 \%$ B. The column and sample tray temperature were kept at $40{ }^{\circ} \mathrm{C}$ and $20^{\circ} \mathrm{C}$, respectively. The mobile phase flow-rate was set $500 \mu \mathrm{L} \mathrm{min}{ }^{-1}$ and the injection volume was $10 \mu \mathrm{L}$.

The MS/MS parameters were optimized individually for each compound by continuous infusion of $1.0 \mu \mathrm{g} \mathrm{mL} \mathrm{L}^{-1}$ standard solution in the initial mobile phase into the mass spectrometer. Instrument parameters were: capillary voltage kept at $-4000 \mathrm{~V}$. Capillary and vaporizer temperature were $220^{\circ} \mathrm{C}$ and $230{ }^{\circ} \mathrm{C}$, respectively. Nitrogen was used as a sheath gas, and auxiliary gas at flow rates of 20 , and 15 arbitrary units, respectively. Argon gas was used as a collisioninduced-dissociation (CID) gas at 1.9 mTorr. The Xcalibur software version 2.0 (Thermo Fisher Scientific) was used to control the instruments and to process data. The selective reaction monitoring (SRM) in negative mode was used. Selected SRM transition, collision energy, tube lens, and retention times for analysis of parabens in baby wipes are shown in Table 1.

\section{Sample preparation}

A total of 50 baby wipes were purchased from July to October 2017 in Brazilian market (supermarket and drugstore). Name of the product (commercial name), expiration date, manufacturer, and sampling location were recorded. All samples were analyzed before the expiration date. it is important to highlight that among the content list of baby wipes samples, only three samples describe parabens in their list (MeP, EtP, PrP and BuP) and 22 samples describe MeP in their list. There is no description about the presence parabens in their list of contents in 25 samples.

Sample extraction for quantification of parabens was performed as previously described by Liao and Kannan with modifications. ${ }^{1}$ Briefly, a square spot $(\sim 2 \mathrm{~cm})$ was taken from the middle of each wipe. The spot was transferred into a polypropylene tube and weighted. After spiking with $10 \mu \mathrm{L}$ of parabens internal standard

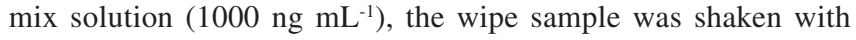
$5 \mathrm{~mL}$ methanol. The extraction of parabens was carried out by two cycles of vortex $(60 \mathrm{~s})$ following ultrasonication for $10 \mathrm{~min}$. After centrifugation at $2500 \times g$ for $10 \mathrm{~min}$, the supernatants were transferred into a new polypropylene tube $(15 \mathrm{~mL})$. The supernatants were combined, evaporated to dryness in a vacuum concentrator. Finally, the residues were reconstituted with $500 \mu \mathrm{L}$ of methanol:water $(1: 1)$, vortexed for $10 \mathrm{~s}$, transferred into a vial and $10 \mu \mathrm{L}$ was injected into the LC-MS/MS for parabens analysis. The samples were kept in their original packaging protected from light and humidity, at room temperature. The sample preparation was performed in the same day of the sample analysis.

\section{Quality control and data analysis}

First, five samples were randomly selected, and then extraction was carried out to analyze the parabens extraction efficiency from baby wipes using previously described method. These samples were re-extracted and re-analyzed. Parabens were not detected after the first extraction, demonstrating that the procedure used was able to extract around $100 \%$ of all parabens from wipes. The identification Parabens was based on the mass transition and the retention time relative to ${ }^{13} \mathrm{C}$-labeled internal standards (Table 1). Quantification of the analytes was based on linear regressions $(r>0.99)$ generated from a 10-point calibration standard at concentrations ranging from 1 to $500 \mathrm{ng} \mathrm{mL}^{-1}$. The standard calibrations were injected daily at the beginning and at the end of the sample analysis. In order to check the interferences and/or contaminations arising from the treatment process, for each batch of 10 samples analyzed, two method blanks, a spiked blank, and a matrix-spiked sample were processed. The results showed that the parabens were below detection limits in method blanks. Dilutions were carried out when the analyte concentration was above the calibration range however when the concentrations of parabens in wipes were too low, the procedure was repeated with a smaller dilution factor. The limit of detection (LOD) is loosely defined as the lowest amount or lowest concentration of the analyte in a sample which can be reliably detected and identified with the method. On the other hand, limit of quantification (LOQ) is defined as the lowest concentration of analyte that can be determined with an acceptable repeatability and trueness. In order to determine both LOD and LOQ, the signal/noise ratio was used, considering the ratio three and ten-fold, respectively. LOQs range from 0.03 to $1.0 \mathrm{ng} \mathrm{g}^{-1}$ and the LODs ranged from 0.01 to $0.30 \mathrm{ng} \mathrm{g}^{-1}$.

Table 1. Selected SRM transition, internal standard used, optimized collision energy $(\mathrm{CE})$, tube lens, and retention times ( $t \mathrm{R})$ for parabens analysis

\begin{tabular}{|c|c|c|c|c|c|}
\hline Compound (internal standard) & Precursor ion & Product Ion & $\mathrm{CE}$ & Tube lens & $t_{\mathrm{R}}(\min )$ \\
\hline $\mathrm{MeP}\left({ }^{13} \mathrm{C}_{6}-\mathrm{MeP}\right)$ & $151(157)$ & $92(98)$ & $22(24)$ & $105(40)$ & $2.5(2.5)$ \\
\hline $\operatorname{EtP}\left({ }^{13} \mathrm{C}_{6}-\mathrm{EtP}\right)$ & $165(171)$ & $92(98)$ & $23(25)$ & $117(46)$ & $3.3(3.3)$ \\
\hline $\operatorname{PrP}\left({ }^{13} \mathrm{C}_{6}-\mathrm{PrP}\right)$ & $179(185)$ & $92(98)$ & $25(36)$ & $103(50)$ & $4.1(4.1)$ \\
\hline $\mathrm{BuP}\left({ }^{13} \mathrm{C}_{6}-\mathrm{BuP}\right)$ & 193 (199) & $92(98)$ & $27(24)$ & $85(55)$ & $4.8(4.8)$ \\
\hline $\mathrm{BzP}\left({ }^{13} \mathrm{C}_{6}-\mathrm{BuP}\right)$ & 227 (199) & $92(98)$ & $27(24)$ & $115(55)$ & $4.7(4.8)$ \\
\hline
\end{tabular}


Data analysis was performed using SPSS software, Version 20, and Microsoft Excel $2013^{\circledR}$. Descriptive statistics were calculated on a wet weight $\left(\mathrm{ng} \mathrm{g}^{-1}\right)$ basis. The concentrations are reported on a nanogram per gram $\left(\mathrm{ng} \mathrm{g}^{-1}\right)$ and concentrations below the LOQ were assigned a value equal to the LOQ divided by the square root of 2. To examine the relationship among parabens, Spearman's correlation was used. All statistical tests were considered significant if the p-value was $<0.05$.

\section{RESULTS AND DISCUSSION}

The concentrations of each paraben and the total concentrations of parabens (sum of five detected parabens: $\Sigma$ PBs) found in Brazilian baby wet wipes are summarized in Table 2 and Figure 1. The four parabens, namely, MeP, EtP, PrP, and BuP were detected together in at least $65 \%$ of the Brazilian baby wet wipes (BBWW). The rank order of detection rate for each selected paraben was: MeP $(100 \%)>\operatorname{PrP}$ $(94 \%)>\mathrm{BuP}(70 \%)>\operatorname{EtP}(66 \%)$. Since the $\mathrm{BzP}$ was not found in any of the samples, it was excluded from statistical analysis. The highest geometric median concentrations were found for $\mathrm{MeP}\left(4914 \mathrm{ng} \mathrm{g}^{-1}\right)$, $\operatorname{PrP}\left(44.1 \mathrm{ng} \mathrm{g}^{-1}\right)$, EtP (16.9 $\left.\mathrm{ng} \mathrm{g}^{-1}\right)$, and BuP (2.5 $\left.\mathrm{ng} \mathrm{g}^{-1}\right)$. $\Sigma \mathrm{PBs}$ concentrations ranged from 16 to $332470 \mathrm{ng} \mathrm{g}^{-1}$, and the geometric mean and median values were 5348 and $4645 \mathrm{ng} \mathrm{g}^{-1}$, respectively.

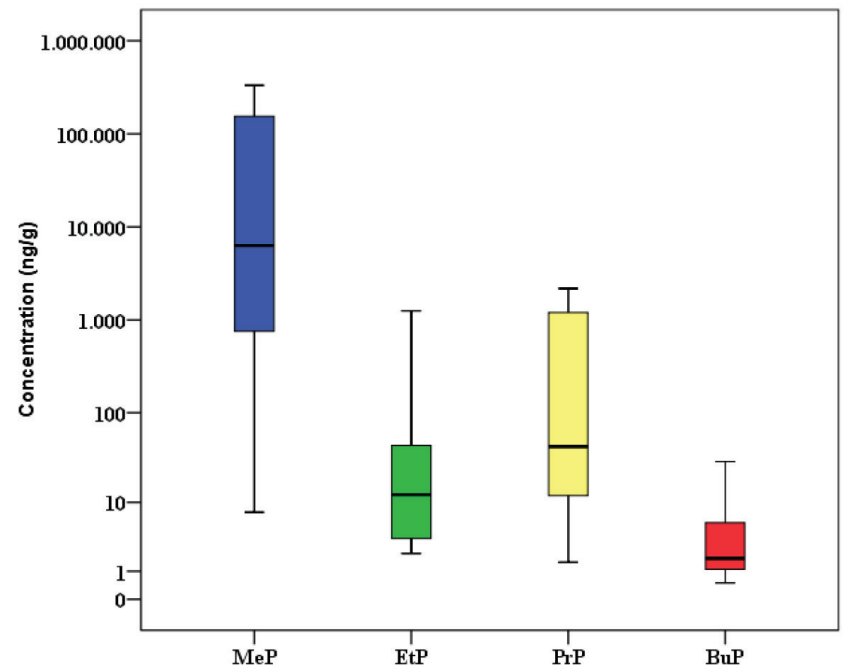

Figure 1. Concentrations of parabens $\left(n g g^{-1}\right)$ in Brazilian baby wet wipes. The horizontal lines represent $10^{\text {th }}, 50^{\text {th }}$ and $90^{\text {th }}$ percentiles and the boxes represent $25^{\text {th }}$ and $75^{\text {th }}$ percentiles

The concentrations of MeP, after logarithmic transformation, were significantly correlated with concentrations of $\operatorname{EtP}(\mathrm{r}=0.535$, $p<0.001)$, and $\operatorname{PrP}(\mathrm{r}=0.671, p<0.001)$. A correlation among $\mathrm{MeP} / \mathrm{EtP}$ and MeP/PrP in BBWW samples suggested that these compounds are used in combination. No significant correlations were found among the concentrations of EtP, PrP and BuP. The size of the alkyl group of parabens influences the antimicrobial activity, the larger the chain, the greater the activity. ${ }^{1,8,14,33}$ Due to the fact of microbial replication occurs in aqueous medium, the use of parabens of higher carbon chain is uncommon in commercial products since an increase in carbon chains decreases its solubility in water. ${ }^{14}$ Thus, short chain parabens such as MeP, EtP and PrP or mixtures of these are the ones most commonly used in commercial products. ${ }^{34,35}$

$\mathrm{MeP}$ is used either alone or with PrP, EtP and/or BuP because of a synergistic effect. Their presence in cosmetic products, especially long-term exposure on a daily basis, may induce adverse health effects. ${ }^{15,25,26}$ Brazilian and European Cosmetics Regulatory Agencies consider the use of parabens as preservatives and name them such as 4-hydroxybenzoic acid and its salts and esters. Since the maximum concentration limits for parabens can be found in the legislation with values of $0.4 \%$ when used as single ester and of $0.8 \%$ when used as mixtures of esters. ${ }^{36,37}$ According to European agency legislation, BuP and $\operatorname{PrP}$ can be used safely in cosmetic products provided that their concentrations do not exceed $0.14 \%$ and are prohibited in the use of cosmetic products for children less than 3 years of age. There are also parabens not allowed for use in cosmetic, such as isopropyl, isobutyl, phenyl, benzyl and pentyl parabens. ${ }^{37}$ In this study, MeP was found in all samples at very high concentration in many of them. The median concentration of $\mathrm{MeP}$ was higher than the maximum concentration permitted by the Brazilian and European regulation. It should be noted that 24 out of 50 samples exceed the permitted value of $0.4 \%$ for $\mathrm{MeP}$ for their use in cosmetics and personal care products. Moreover, the detection rate of PrP and BuP were 94 and $70 \%$, respectively. Our studies suggest that Brazilian babies may be exposed to parabens and its harmful effects through dermal absorption.

\section{Estimation of daily intake (EDI)}

In addition to other sources, absorption of parabens via skin from the uses of wipes seems to be a relevant route of exposure. Considering the high concentrations of parabens found in BBWW and dermal absorption coefficient of parabens, the use of these wipes may constitute an important source of exposure to babies. The evaluation of the quantity and frequency of use of wipes over a day is still scarce. However, the frequency of use can be estimated by a specialist considering that a wiping with moist wipes is performed with each diaper change, which results in an average of five times of use per day. Moist wipes for commercial babies weigh approximately $5 \mathrm{~g}$ per wipe. During the hygiene of the baby, contact with the dermis is not permanent and not all the surface of the wipe is used, therefore, a retention factor should be applied. ${ }^{38}$ According to de van Engelen, ${ }^{39}$ each time a baby wipe is used, there is a dermal contact of $0.5 \mathrm{~mL}$ of liquid with the skin. If $5 \mathrm{~g}$ of wipes are used five times a day and considering the retention factor 0.1 , the final value on the skin will be $2.5 \mathrm{~g}$ /day. Thus, dermal intakes of parabens could be calculated on the basis of equation: ${ }^{40}$

$$
E D I=C \times A \times F \times R f \times \frac{D A}{w^{-l}}
$$

Table 2. Concentrations of parabens $\left(\mathrm{ng} \mathrm{g}^{-1}\right)$ in baby wet wipes from Brazil

\begin{tabular}{|c|c|c|c|c|c|c|}
\hline Baby wipes $(\mathrm{n}=50)$ & $\mathrm{MeP}$ & EtP & $\operatorname{PrP}$ & $\mathrm{BuP}$ & $\mathrm{BzP}$ & $\Sigma \mathrm{PBs}$ \\
\hline$\overline{\mathrm{GM}}$ & 4914 & 16.9 & 44.1 & 2.5 & nd & 5348 \\
\hline Median & 3709 & 16.0 & 37.7 & 1.5 & nd & 4645 \\
\hline Minimum & 7.6 & 2.1 & 0.7 & 0.3 & nd & 16 \\
\hline Maximum & 332237 & 1251 & 2175 & 238 & nd & 332470 \\
\hline Frequency $(\%)$ & 100 & 66 & 94 & 70 & nd & 100 \\
\hline
\end{tabular}

Nd: not detected. 
where EDI is estimated daily intake adjusted for body weight

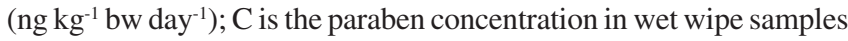
$\left(\mathrm{ng} \mathrm{g}^{-1}\right) ; \mathrm{A}$ is amount of product applied pert time and was assumed to be $5 \mathrm{~g} ; \mathrm{F}$ is frequency of use and was assumed to be 5 events/day; $\mathrm{Rf}$ is retention factor (0.1); DA is the absorption fraction; and $\mathrm{w}$ is average body weight of a boy of 1.0-year-old child of $11.5 \mathrm{~kg}$ ( $\mathrm{kg} \mathrm{bw})$. For C, the geometric mean and 95th percentile concentrations of parabens were used for average and high exposure scenarios, respectively. For DA, as the dermal absorption of parabens, we used a value of $36 \%$, $55 \%, 37 \%$ and $42 \%$ for MeP, EtP, PrP and BuP, respectively in our calculation. ${ }^{38,40}$

Estimated daily intake adjusted for body weight of parabens and sum of them are shown in Table 3. The highest EDI values were found for MeP, followed by PrP, EtP, and BuP. The median EDIs of MeP, PrP, EtP, and BuP were 385, 3.5, 2.0, and $0.23 \mathrm{ng} \mathrm{kg}^{-1}$ bw day ${ }^{-1}$, respectively. The EDI values found for parabens from baby wipes were below the acceptable daily intake (ADI) of $0-10 \mathrm{mg} \mathrm{kg}^{-1}$ bw day for MeP, EtP and their sodium salts, according to the European Food Safety Authority's recommendations. ${ }^{41}$

Table 3. Estimated daily intake adjusted for body weight $\left(\mathrm{ng} \mathrm{kg}^{-1}\right.$ bw day-1) of each and total parabens via uses of baby wet wipes by the babies of 1 year old $(11.5 \mathrm{~kg})$

\begin{tabular}{lcc}
\hline Parabens & Median & $95^{\text {th }}$ Percentile \\
\hline MeP & 385 & 24,876 \\
EtP & 2.0 & 144 \\
PrP & 3.5 & 168 \\
BuP & 0.23 & 21 \\
\hline Iparabens & 391 & 25,209 \\
\hline
\end{tabular}

However, the EDI values found for PrP in the current study opposed the recommendation of European Food Safety Authority (EFSA). EFSA's panel evaluated the parabens' toxicology and the safety of their usage in foods and established an ADI of $0-10 \mathrm{mg} \mathrm{kg}^{-1}$ bw for MeP and EtP and their sodium salts. However, the panel was unable to recommend an ADI for PrP because this specific paraben had endocrine disrupts effect at a low dose in male rats and also the lack of a clear No Observed Adverse Effect Level (NOAEL) for this effect. $^{42}$

\section{CONCLUSIONS}

Indeed, its clearly the negligence regarding the human exposure to parabens through commercial baby wipes in comparison with the other sources such as food, cosmetics, and pharmaceuticals. Although low concentrations were measured in some parabens categories (MeP and EtP), we were able to identify concentrations of parabens' classes which are prohibited in cosmetics designed for application on the nappy area of children below three years, $\operatorname{PrP}$ and BuP have shown a detection rate of 94 and $70 \%$, respectively. The outcomes hereby bright the literature gap regarding the parabens exposure in commercial baby wipes including two parabens which are prohibited in cosmetic products for children under three years of age ( $\mathrm{PrP}$ and $\mathrm{BuP}$ ) and these milestones will serve as a base for future studies on commercial cosmetic products designed for application on children and babies.

\section{ACKNOWLEDGMENTS}

This research was supported by National Institute of Science \& Technology in Bioanalytics (INCTBio CNPq 434303/2016-0),
São Paulo Research foundation (Fundação de Amparo à Pesquisa do Estado de São Paulo-FAPESP, Process numbers: 2016/02949-6, 2014/19914-5, 2014/50867-3, 2013/23710-3), and Coordenação de Aperfeiçoamento de Pessoal de Nível Superior - Brasil (CAPES, finance code, 001). Thanks for financial support and for granting research fellowships.

\section{REFERENCES}

1. Liao, C.; Kannan, K.; Sci. Total Environ. 2014, 475, 8.

2. Chung, B. Y.; Choi, S. M.; Roh, T. H.; Lim, D. S.; Ahn, M. Y.; Kim, Y. J.; Kim, H. S.; Lee, B. M.; J. Toxicol. Environ. Health, Part A 2019, 82, 351 .

3. Diamanti-Kandarakis, E.; Bourguignon, J. P.; Giudice, L. C.; Hauser, R.; Prins, G. S.; Soto, A. M.; Zoeller, R. T.; Gore, A. C.; Endocr. Rev. 2009, 30, 293.

4. Jiménez-Díaz, I.; Vela-Soria, F.; Rodríguez-Gómez, R.; Zafra-Gómez, A.; Ballesteros, O.; Navalón, A.; Anal. Chim. Acta 2015, 892, 27.

5. Rocha, B. A.; Asimakopoulos, A. G.; Barbosa, F.; Kannan, K.; Sci. Total. Environ. 2017, 586, 152.

6. Rocha, B. A.; de Oliveira, A. R. M.; Barbosa, F.; Talanta 2018, 183, 94.

7. Rocha, B. A.; Asimakopoulos, A. G.; Honda, M.; da Costa, N. L.; Barbosa, R. M.; Barbosa, F.; Kannan, K.; Environ. Int. 2018, 116, 269.

8. Liao, C.; Chen, L.; Kannan, K.; Environ. Int. 2013, 57, 68.

9. Guo, Y.; Kannan, K.; Environ. Sci. Technol. 2013, 47, 1444.

10. Guo, Y.; Wang, L.; Kannan, K.; Arch. Environ. Contam. Toxicol. 2013, 66, 113.

11. Casals-Casas, C.; Desvergne, B.; Annu. Rev. Physiol. 2011, 73, 135.

12. Barrios-Estrada, C.; de Jesús Rostro-Alanis, M.; Muñoz-Gutiérrez, B. D.; Iqbal, H. M. N.; Kannan, S.; Parra-Saldívar, R.; Sci. Total Environ. 2018, 612, 1516.

13. Błędzka, D.; Gromadzińska, J.; Wąsowicz, W.; Environ. Int. 2014, 67, 27.

14. Soni, M. G.; Carabin, I. G.; Burdock, G. A.; Food Chem. Toxicol. 2005, 43,985 .

15. Boberg, J.; Taxvig, C.; Christiansen, S.; Hass, U.; Reprod. Toxicol. 2010, 30,301 .

16. Dodge, L. E.; Kelley, K. E.; Williams, P. L.; Williams, M. A.; Hernandez-Diaz, S.; Missmer, S. A.; Hauser, R.; Reprod. Toxicol. 2015, $52,93$.

17. Mbah, C. J.; Pharmazie 2007, 62, 351.

18. Ye, X.; Kuklenyik, Z.; Bishop, A.; Needham, L., Calafat, A.; J. Chromatogr. B 2006, 844, 53.

19. Lakeram, M.; Lockley, D. J.; Sanders, D. J.; Pendlington, R.; Forbes, B.; J. Biomol. Screening 2007, 12, 84.

20. Sandanger, T. M.; Huber, S.; Moe, M. K.; Braathen, T.; Leknes, H.; Lund, E.; J. Expo. Sci. Environ. Epidemiol. 2011, 21, 595.

21. Engeli, R. T.; Rohrer, S. R.; Vuorinen, A.; Herdlinger, S.; Kaserer, T.; Leugger, S.; Schuster, D.; Odermatt, A.; Int. J. Mol. Sci. 2017, 18, 2007.

22. El Hussein, S.; Muret, P.; Berard, M.; Makki, S.; Humbert, P. Exp. Dermatol. 2007, 16, 830 .

23. Abbas, S.; Greige-Gerges, H.; Karam, N.; Piet, M. H.; Netter, P.; Magdalou, J. Drug Metab. Pharmacokinet. 2010, 25, 568.

24. Oishi, S.; Arch. Toxicol. 2002, 76, 423.

25. Darbre, P. D.; Harvey, P. W.; J. Appl. Toxicol. 2008, 28, 561.

26. Darbre, P. D.; Aljarrah, A.; Miller, W. R.; Coldham, N.G.; Sauer, M. J.; Pope, G. S.; J. Appl. Toxicol. 2004, 24, 5.

27. Witorsch, R. J.; Thomas, J. A.; Crit. Rev. Toxicol. 2010, 40, 1.

28. Jiménez-Díaz, I.; Vela-Soria, F.; Rodríguez-Gómez, R.; Zafra-Gómez, A.; Ballesteros, O.; Navalón, A.; Anal. Chim. Acta 2015, 892, 27.

29. Angerer, J.; Bernauer, U.; Chambers, C.; Chaudhry, Q.; Degen, G.; Nielsen, E.; Platzek, T.; Rastogi, S. C.; Rogiers, V.; Rousselle, C.; Sanner, T.; Vanbenthem, J.; Vanen-Gelen, J.; Vinardell, M. P.; Waring, 
R.; White, I. R. 2011. SCCS/1446/11:SCCS (Scientific Committee On Consumer Safety). Clarification on opinion the light of the Danish clause of safe guard banning the use of parabens in cosmetic products intended for children under three years of age, 10 october 2011. DOI: $10.2772 / 20395$.

30. Bernauer, U.; Chaudhry, Q.; Degen, G.; Nielsen, E.; Platzek, T.; Rastogi, S. C.; Rousselle, C.; Vanbenthem, J.; Coenraads, P.; Dusinska, M.; Gawkrodger, D.; Lilienblum, W.; Luch, A.; Metzler, M.; MonteiroRivière, N. 2013. SCCS/1514/13:SCCS (SCIENTIFIC COMMITTEE ON CONSUMER SAFETY). Opinion on parabens, 3 MAY 2013. DOI:10. 2772/66369.

31. Aschenbeck, K. A.; Warshaw, E. M.; Dermatitis 2017, 28, 353.

32. Cashman, A. L.; Warshaw, E. M.; Dermatitis 2005, 16, 57.

33. Taxvig, C.; Vinggaard, A. M.; Hass, U.; Axelstad, M.; Boberg, J.; Hansen, P. R.; Frederiksen, H.; Nellemann. C.;. Toxicol. Sci. 2008, 106, 206.

34. Andersen, F. A.; Int. J. Toxicol. 2008, 27, 1.
35. Eriksson, E.; Andersen, H. R.; Ledin, A.; J. Hazard Mater. 2008, 156 , 240.

36. Anvisa - Brazilian Health Surveillance Agency, Brazil, 2000. Resolução No -79

37. European Commission Regulation (EU) No 1004/2014 of 18 September 2014 amending Annex V to Regulation (EC) No 1223/2009 of the European Parliament and of the Council on cosmetic productsRegulation (EC) No 1223/2009 of the European Parliament and of the Council of 30 November 2009 on cosmetic products (recast), in Off. J. Eur. Union L342/59., 2009.

38. Goossens, A.; Current Treatment Options in Allergy 2015, 2, 228.

39. van Engelen, J. G. M.; de Lodder, L. C. H. P.; RIVM Report 2004, 1.

40. Gosens, I.; Delmaar CJE, Ter Burg W, De Heer C, Schuur AG. J. Expo. Sci. Environ. Epidemiol. 2014, 24, 208.

41. European Food Safety Authority (EFSA); EFSA Journal 2004, 2, 84.

42. Oishi, S.; Food Chem. Toxicol. 2002, 40, 1807. 